\title{
EBULLITION DOMESTIQUE ET TENEUR EN RIBOFLAVINE DU LAIT DE VACHE
}

\author{
par \\ JEAN CAUSERET
}

Avec la collaboration technique d'EveLyNE CIROT

\section{I. - INTRODUCTION}

La riboflavine est la principale vitamine du lait de vache. Elle est y présente à un taux compris généralement entre 1 et 3 milligrammes par litre, avec une moyenne voisine de 1 mgr. 8 . Il s'agit là de valeurs élevées, puisque le besoin de l'adulte moyen se situe vraisemblablement aux alentours de 2 milligrammes par jour.

L'influence de l'ébullition domestique du lait sur le sort de la riboflavine a été peu étudiée. EscuDERo et collab. [2], - se basant uniquement sur des essais de croissance effectués durant six semaines sur de jeunes rats soumis à un régime de base dépourvu de vitamines du complexe $B$ et complété par des quantités égales de lait cru ou bouilli ont affirmé que, dans leur ensemble, ces vitamines ne sont pas atteintes par l'ébullition domestique; mais la nature du critère qu'ils ont employé ne justifie guère une conclusion aussi formelle. Seul, à notre connaissance, KNAUT [3] a dosé comparativement la riboflavine dans un même lait cru ou bouilli (ébullition de 2 minutes) : les quantités trouvées ont été les mêmes dans les deux cas.

Nous avons donc entrepris une étude systématique de la question, en cherchant dans une certaine mesure à tenir compte des conditions diverses que l'on peut rencontrer dans la pratique : utilisation ou rejet de la "peau " qui se forme à la surface du lait, perte inévitable du "gratin " qui adhère à la casserole, consommation du lait aussitôt après l'ébullition ou après un long repos, souvent à la température de la pièce.

\section{II. - PROTOCOLE EXPÉRIMENTAL}

N'ayant pas la possibilité de déterminer simultanément les taux de riboflavine d'un lait non bouilli, d'un même lait bouilli, de la " peau » et du "gratin ) correspondants, nous avons effectué notre travail en trois temps :

- détermination des pertes de riboflavine dans la "peau";

- détermination des pertes dans le "gratin ";

- détermination comparative des teneurs en riboflavine d'un lait non bouilli et bouilli, la "peau " et le "gratin " étant éliminés. 
Les prélèvements ont été effectués une demi-heure après l'ébullition, ainsi que 24 heures après dans un certain nombre de cas.

\section{Conditions d'ébullition}

Toutes les opérations ont été effectuées dans les conditions suivantes, voisines de celles utilisées par Plommet [6].

Deux litres de lait pasteurisé conditionné $\left(^{\mathbf{1}}\right)$, portant la date du jour et titrant le plus souvent 17-19० D., sont mélangés. On prélève sur le mélange deux fractions de 900 millilitres, qui sont versées chacune dans une casserole d'aluminium (diamètre : $160 \mathrm{~mm}$.) et chauffées sur un réchaud à gaz jusqu'à la montée du lait : celle-ci se produit après 10 ou 11 minutes, la température du liquide atteignant alors $99^{\circ} \mathrm{C}$.. On laisse ensuite le lait refroidir spontanément à l'air libre.

Une demi-heure après, les prélèvements de peau, de gratin ou de lait sont effectués dans l'une des casseroles, l'autre étant couverte et déposée pendant 24 heures dans une salle dont la température atteint $20^{\circ} \mathrm{C}$.; puis des prélèvements comparables sont effectués dans cette seconde casserole.

Les 200 millilitres de lait non bouilli sont utilisés immédiatement pour le dosage de la riboflavine.

\section{Prélèvements}

Chaque casserole est pesée vide (poids : $P_{1}$ ), puis lorsqu'elle est remplie de lait bouilli (poids : $\mathrm{P}_{2}$ ). La peau est prélevée au moyen d'une écumoire et égouttée rapidement au-dessus de la casserole, qui est ensuite pesée à nouveau (poids : $P_{3}$ ). Après transvasement du lait, la casserole ne renfermant plus que le gratin est pesée une dernière fois (poids : $P_{4}$ ) et le gratin est recueilli au moyen d'une spatule.

$$
\begin{aligned}
& \text { Poids de la peau : } \mathrm{P}_{2}-\mathrm{P}_{3} \\
& \text { Poids du gratin : } \mathrm{P}_{4}-\mathrm{P}_{1} \\
& \text { Poids du lait bouilli : } \mathrm{P}_{3}-\mathrm{P}_{4}
\end{aligned}
$$

\section{Dosage de la riboflavine}

La peau et le gratin sont homogénisés séparément dans deux mortiers.

Les prises d'essai sont respectivement de 10 millilitres pour le lait, de 3 à 5 grammes pour la peau et le gratin.

(1) Bien que l'ébullition du lait correctement pasteurisé et conditionné en caraf es soit inutile, elle demeure couramment pratiquée en France [6]. 
La vitamine est extraite à l'acétone acide (1). Après destruction des impuretés gênantes par oxydation permanganique, la fluorescence de l'extrait est déterminée au spectrophotomètre. Avec quelques variantes, la méthode employée est celle de l'Association of Vitamin Chemists [1].

\section{III. - RÉSULTATS}

\section{$1^{0}$ Teneur du lait en riboflavine.}

Vingt et un échantillons de lait pasteurisé conditionné ont été analysés, entre mars et août de la même année. Ils contenaient en moyenne 2 mgr. 0 de riboflavine par litre, valeur qui correspond à celle qu'on relève dans la plupart des Tables de Composition des Aliments pour le lait cru ou pasteurisé.

Les variations du taux de riboflavine sont considérables, puisque les valeurs extrêmes atteignent 1 mgr. 2 et 2 mgr. 5 ; toutefois, 16 valeurs sur 21 sont comprises dans l'intervalle de $1 \mathrm{mgr}$. 6 à 2 mgr. 4. On notera l'absence de variation nette avec la saison $\left({ }^{2}\right)$.

\section{Perte de riboflavine dans la "peau ".}

Dans les conditions décrites plus haut, les quantités de "peau » recueillies atteignent en moyenne 11 grammes par litre, 30 minutes après l'ébullition, et 19 grạmmes, 24 heures après. Dans des conditions pourtant assez proches, Plommet avait obtenu 10 grammes de peau, 30 minutes après l'ébullition, et 58 grammes, 24 heures après.

Bien que la peau apparaisse plus riche en riboflavine que le lait, la perte de riboflavine qui résulte de son rejet est pratiquement négligeable (tableau I).

\section{$3^{\circ}$ Perte de riboflavine dans le « gratin ».}

Les quantités de gratin recueillies atteignent en moyenne 9 grammes par litre, 30 minutes après l'ébullition, et 13 grammes par litre, 24 heures après. Plommet en avait obtenu $16-17$ gr. 5 .

Comme la peau, le gratin semble plus riche en riboflavine que le lait, mais son élimination n'entraîne qu'une perte de vitamines également négligeable (tableau II).

\section{$4^{\circ}$ Teneurs comparées du lait non bouilli et du lait bouilli en riboflavine.}

La perte de poids due à l'évaporation au cours de l'ébullition

(1) On ne pratique pas de digestion enzymatique, des essais préliminaires aveo takadiastase et papaine ayant montré que les résultats obtenus n'en sont pas sensiblement modifiés.

(2) C'est en hiver seulement que Javillier [4] et Kon [5] ont signalé un net abaissement du taux de riboflavine du lait. 
TABLEAU I

TENEUR EN RIBOFLAVINE

DE LA " PEAU " REGUEILLIE SUR LE LAIT BOUILLI

\begin{tabular}{|c|c|c|c|c|c|}
\hline & ${ }^{*}$ & & Riboflavine dans & $\begin{array}{r}\text { Riboflavine dans } \\
\text { sur } 1 \text { litre de }\end{array}$ & $\begin{array}{l}\text { la peau recueillie } \\
\text { lait bouilli }\end{array}$ \\
\hline Echan & ntillon & $\begin{array}{l}\text { Duree du repos } \\
\text { après ébullition }\end{array}$ & $\begin{array}{c}\text { non bouilli } \\
\mu \mathrm{gr} .\end{array}$ & $\begin{array}{c}\text { en valeur absolue } \\
\mu \mathrm{gr} .\end{array}$ & $\begin{array}{c}\text { perte } \\
\text { correspondante } \\
\%\end{array}$ \\
\hline 1 (avril) & $\ldots \ldots$ & 0 h. 30 & 2.050 & 35 & 1,7 \\
\hline 2 (avril) & $\ldots \ldots$ & 0 h. 30 & 1.565 & 29 & 1,8 \\
\hline 3 (avril) & $\ldots \ldots$ & 0 h. 30 & 2.420 & 51 & 2,1 \\
\hline 4 (mai) & $\ldots \ldots$ & 0 h.' 30 & 1.370 & 37 & 2,7 \\
\hline Moyenr & nes .... & & 1.851 & 38 & 2,0 \\
\hline I (avril) & $\ldots \ldots$ & 24 heures & 2.050 & 39 & 1,9 \\
\hline 2 (avril) & $\ldots \ldots$ & . 24 heures & 1.565 & $2 i$ & 1,2 \\
\hline 3 (avril) & $\ldots \ldots$ & 24 heures & 2.420 & 66 & 2,7 \\
\hline 4 (mai) & $\ldots \ldots$ & 24 heures & 1.370 & 53 & 3,8 \\
\hline Moyenr & nes ..... & & 1.851 & 45 & 2,4 \\
\hline
\end{tabular}

TABLEAU II

TENEUR EN RIBOFLAVINE DU " GRATIN "

\begin{tabular}{|c|c|c|c|c|c|}
\hline & & & Riboflavine dans & $\begin{array}{l}\text { Riboflavine dan } \\
\text { à partir de } 1 \text { litr }\end{array}$ & $\begin{array}{l}\text { le gratin formé } \\
\text { de lait bouilli }\end{array}$ \\
\hline Ech & ntillon & après ébullition & non houilli & . & perte \\
\hline 5 (mai) & $\ldots \ldots$ & $0 \mathrm{~h} .30$ & 2.310 & 34 & 1,5 \\
\hline 6 (mai) & $\ldots \ldots$ & 0 h. 30 & 1.657 & 31 & 1,9 \\
\hline 7 (mai) & $\ldots \ldots$ & 0 h. 30 & 2.047 & 41 & 2,0 \\
\hline 8 (mai) & $\ldots \ldots$ & 0 h. 30 & 1.758 & 30 & 1,7 \\
\hline Moyer & nes .... & & 1. 943 & 34 & 1,7 \\
\hline 5 (mai) & $\ldots \ldots$ & 24 heures & 2.310 & 40 & 1,7 \\
\hline 6 (mai) & $\ldots \ldots$ & 24 heures & 1.657 & 48 & 2,9 \\
\hline 7 (mai) & $\ldots \ldots$ & 24 heures & 2.047 & 48 & 2,3 \\
\hline 8 (mai) & $\ldots \ldots$ & 24 heures & 1.758 & 37 & 2,1 \\
\hline Moyer & nes .... & & 1.943 & 43 & 2,2 \\
\hline
\end{tabular}

est en moyenne de 40 grammes par litre, 30 minutes après l'ébullition, et de 60 grammes, 24 heures après. Ces valeurs sont inférieures à celles de Plommet (50 et 80 gr.). 
$3^{\circ}$ Le rejet de la "peau " et la formation d'un "gratin " sur les parois $\mathrm{du}$ récipient où le lait a bouilli ne sont pas responsables de pertes de riboflavine importantes (moins de $5 \%$ ).

(Laboratoire d'Etudes sur la Nutrition de l'I. N. R. A.,' Paris.)

\section{REFERENCES BIBLIOGRAPHIQUES}

[1] Association of vitamin chemists. Methods of Vitamin Assay. Interscience Publ., London, 1947.

[2] A. Escudero, M. L. Herraiz et H. G. De Alvarez Herrero. Rev. Dietologia, 1944, 2, 199.

[3] T. Knaut. Rocz. Nauk rol. (B), 1955, 70, 197.

[4] M. Javilliter. C. R. Ac, Agric., 1940, 26, 391.

[5] K. S. Kon. Chem. Ind., 1943, 62, 478.

[6] M. Plommet. Ann. Technol. Agric., 1956, 4, 493.

\section{L'INDUSTRIE LAITIËRE EN ITALIE}

par

le Docteur Giovanni DELFORNO

Institut Zootechnique et Fromager du Piémont (Turin)

La variété des terrains et des climats a créé et entretenu les caractéristiques de l'industrie laitière en Italie.

Avant l'apparition des techniques modernes et des moyens commerciaux actuellement mis en œuvre, permettant en particulier la valorisation des sous-produits, la production du lait en ce Pays était en relation directe avec les besoins de la consommation locale ; elle a dû sa rapide et considérable extension au développement et au perfectionnement industriels.

Sur un peu plus de 90.388.000 hectolitres de lait, produits en 1957, presque la moitié, soit 41.553 .000 hectolitres, ont été transformés en fromage, tandis que 26.893.000 hectolitres environ étaient destinés à la consommation en nature. Danș ces conditions, on a pu compter, en cette même année, 3.360 .000 quintaux de fromages et 598.000 quintaux de beurre, avec, sans les dénombrer ici, un grand nombre d'autres produits et sous-produits.

Comme on le voit, les fabrications fromagères constituent l'essentiel et il en fut toujours de même dans le passé.

Les plus anciens renseignements, dont on dispose, ont trait à l'époque où les Etrusques et les Ombriens s'établirent dans les Alpes Réthiques, d'où ils se répandirent dans la plaine du Pô jusqu'en Emilie. Nombreux sont les témoignages sur les anoiens fromages italiens, laissés par les Auteurs latins. 
non bouilli. Cette différence n'est pas significative, ce qui conduit à conclure, compte tenu aussi de la présence de $4 \%$ environ de la vitamine totale dans la peau et le gratin, qu'une ébullition domestique de courte durée respecte généralement la riboflavine du lait.

Toutefois, la variabilité des résultats obtenus ne permet pas une conclusion aussi formelle. Les cas extrêmes correspondent à une perte vitaminique de $30 \%$ et à un gain de $14 \%$; dans 5 cas sur 13 , la perte dépasse $15 \%$. Même en admettant que les résultats obtenus puissent être affectés d'une erreur relative de $10 \%$, une perte de vitamine appréciable s'est donc produite dans plusieurs cas, et un gain apparent dans un cas au moins.

Cinq échantillons seulement on fait l'objet de déterminations 24 heures après l'ébullition. Pour trois d'entre eux (nos 10, 20 et $21)$, les résultats obtenus sont du même ordre de grandeur qu'après repos d'une demi-heure. Pour les deux autres (nos 14 et 19), un gain apparent de riboflavine pendant le repos du lait s'est produit, le taux vitaminique passant respectivement de 1,58 à $2 \mathrm{mgr}$. 00 et de 2,09 à 2 mgr. 33 .

La signification de l'accroissement apparent de la teneur du lait en vitamine $B_{2}$ est difficile à indiquer. Cet accroissement pourrait en principe résulter de la libération de formes liées de la riboflavine. Cependant, on sait que le lait contient peu de riboflavine sous forme nucléotidique, et nous avons rappelé que l'action combinée d'une extraction par l'acétone acide et d'une digestion par la takadiastase et la papaïne ne nous a jamais conduits, avec le lait pasteurisé non bouilli, à des résultats plus élevés que la seule extraction par l'acétone acide.

\section{IV. - RÉSUMÉ ET CONCLUSIONS}

$1^{0}$ La riboflavine a été dosée de mars à août de la même année, dans 21 échantillons de lait pasteurisé conditionné du commerce. $80 \%$ des valeurs obtenues étaient comprises entre 1,6 et $2 \mathrm{mgr} .4$ par litre, la moyenne générale étant de $2 \mathrm{mgr}$. 0 . Aucune variation saisonnière n'a pu être mise en évidence.

$2^{\circ}$ La riboflavine a également été dosée dans une partie des échantillons après ébullition - interrompue à la montée du lait suivie d'un repos d'une demi-heure ou de 24 heures. Dans la majeure partie des cas, ce traitement n'a pas exercé d'effet sensible sur la vitamine. Toutefois, dans le lait analysé une demi-heure après l'ébullition, on a observé dans quelques cas une perte de riboflavine pouvant aller, compte tenu de la quantité retrouvée dans la "peau" et le "gratin ", jusqu'à $25 \%$. Dans le lait analysé après 24 heures de repos, on a retrouvé, soit le même taux de vitamine, soit un taux un peu plus élevé. 
$3^{0}$ Le rejet de la "peau " et la formation d'un "gratin » sur les parois du récipient où le lait a bouilli ne sont pas responsables de pertes de riboflavine importantes (moins de $5 \%$ ).

(Laboratoire d'Etudes sur la Nutrition de l'I. N. R. A., Paris.)

\title{
REFERENCES BIBLIOGRAPHIQUES
}

[1] Association of vitamin chemists. Methods of Vitamin Assay. Interscience Publ., London, 1947.

[2] A. Escudero, M. L. Herraiz et H. G. De Alvarez Herrero. Rev. Dietologia, 1944, 2, 199.

[3] T. Knaut. Rocz. Nauk rol. (B), 1955, 70, 197.

[4] M. Javillier. C. R. Ac. Agric., 1940, 26, 391.

[5] K. S. Kon. Chem. Ind., 1943, 62, 478.

[6] M. Plommet. Ann. Technol. Agric., 1956, 4, 493.

\section{L'INDUSTRIE LAITIÈRE EN ITALIE}

par

\author{
le Docteur Giovanni DELFORNO
}

Institut Zootechnique et Fromager du Piémont (Turin)

La variété des terrains et des climats a créé et entretenu les caractéristiques de l'industrie laitière en Italie.

Avant l'apparition des techniques modernes et des moyens commerciaux actuellement mis en œuvre, permettant en particulier la valorisation des sous-produits, la production du lait en ce Pays était en relation directe avec les besoins de la consommation locale ; elle a dû sa rapide et considérable extension au développement et au perfectionnement industriels.

Sur un peu plus de 90.388.000 hectolitres de lait, produits en 1957, presque la moitié, soit 41.553 .000 hectolitres, ont été transformés en fromage, tandis que 26.893.000 hectolitres environ étaient destinés à la consommation en nature. Danș ces conditions, on a pu compter, en cette même année, 3.360 .000 quintaux de fromages et 598.000 quintaux de beurre, avec, sans les dénombrer ici, un grand nombre d'autres produits et sous-produits.

Comme on le voit, les fabrications fromagères constituent l'essentiel et il en fut toujours de même dans le passé.

Les plus anciens renseignements, dont on dispose, ont trait à l'époque où les Etrusques et les Ombriens s'établirent dans les Alpes Réthiques, d'où ils se répandirent dans la plaine du Pô jusqu'en Emilie. Nombreux sont les témoignages sur les anciens fromages italiens, laissés par les Auteurs latins. 